\title{
Differential effects of the two amino acid sensing systems, the GCN2 kinase and the mTOR complex 1, on primary human alloreactive $\mathrm{CD4}^{+} \mathrm{T}$-cells
}

\author{
THEODOROS ELEFTHERIADIS, GEORGIOS PISSAS, GEORGIA ANTONIADI, VASSILIOS LIAKOPOULOS, \\ KONSTANTINA TSOGKA, MARIA SOUNIDAKI and IOANNIS STEFANIDIS
}

Department of Nephrology, Medical School, University of Thessaly, 41110 Larissa, Greece

Received December 16, 2015; Accepted March 11, 2016

DOI: $10.3892 /$ ijmm.2016.2547

\begin{abstract}
Amino acid deprivation activates general control nonderepressible 2 (GCN2) kinase and inhibits mammalian target of rapamycin (mTOR), affecting the immune response. In this study, the effects of GCN2 kinase activation or mTOR inhibition on human alloreactive $\mathrm{CD} 4^{+} \mathrm{T}$-cells were evaluated. The mixed lymphocyte reaction, as a model of alloreactivity, the GCN2 kinase activator, tryptophanol (TRP), and the mTOR complex 1 inhibitor, rapamycin (RAP), were used. Both TRP and RAP suppressed cell proliferation and induced cell apoptosis. These events were p53-independent in the case of RAP, but were accompanied by an increase in p53 levels in the case of TRP. TRP decreased the levels of the Th2 signature transcription factor, GATA-3, as RAP did, yet the latter also decreased the levels of the Th1 and Th17 signature transcription factors, T-bet and ROR $\gamma \mathrm{t}$, whereas it increased the levels of the Treg signature transcription factor, FoxP3. Accordingly, TRP decreased the production of interleukin (IL)-4, as RAP did, but RAP also decreased the levels of interferon- $\gamma$ (IFN- $\gamma$ ) and IL-17. Both TRP and RAP increased the levels of IL-10. As regards hypoxia-inducible factor- $1 \alpha(\mathrm{HIF}-1 \alpha)$, which upregulates the Th17/Treg ratio, its levels were decreased by RAP. TRP increased the HIF-1 $\alpha$ levels, which however, remained inactive. In conclusion, our findings indicate that, in primary human alloreactive $\mathrm{CD}^{+}{ }^{+} \mathrm{T}$-cells, the two systems that sense amino acid deprivation affect cell proliferation, apoptosis and differentiation in different ways or through different mechanisms. Both mTOR inhibition and GCN2 kinase activation exert immunosuppressive effects, since they inhibit cell proliferation and induce apoptosis. As regards $\mathrm{CD} 4^{+} \mathrm{T}$-cell differentiation, mTOR inhibition exerted a more profound effect, since it suppressed differentiation into the Th1, Th2 and Th17 lineages,
\end{abstract}

Correspondence to: Professor Theodoros Eleftheriadis, Department of Nephrology, Medical School, University of Thessaly, Neo Ktirio, Mezourlo Hill, 41110 Larissa, Greece

E-mail: teleftheriadis@yahoo.com

Key words: T-cell, general control nonderepressible 2 kinase, mammalian target of rapamycin, proliferation, apoptosis, differentiation while it induced Treg differentiation. On the contrary, the activation of GCN2 kinase suppressed only Th2 differentiation.

\section{Introduction}

During the immune response, amino acid deprivation constitutes a significant immunoregulatory mechanism. There are certain enzymes, such as arginase I in myeloid-derived suppressor cells and indoleamine 2,3-dioxygenase (IDO) in antigen-presenting cells that cause the depletion of certain amino acids and suppress T-cell effector function $(1,2)$.

In eukaryotic cells, there are two conservative mechanisms that sense amino acid deprivation. The first is able to sense the lack of any amino acid and is based on the activation of the general control nonderepressible 2 (GCN2) kinase by uncharged tRNA $(3,4)$. The second includes the inhibition of the mammalian target of rapamycin complex 1 (mTORC1), which, in the presence of amino acids, remains active through a complex mechanism that recruits $\mathrm{mTORC} 1$ to the outer lysosomal membrane where it interacts with its activator, Rheb (4-6). There are data supporting that mTORC1 is sensitive to the depletion of certain amino acids and more precisely to leucine, isoleucine, valine and possibly arginine (4). Indeed, as previously demonstrated, in a model of alloreactivity, IDO-induced tryptophan depletion activated the GCN2 kinase, but did not affect mTORC1 activity in primary human CD4+ T-cells (7).

Upon amino acid deprivation, both systems downregulate global protein translation, while they enhance the translation of certain proteins, again through different mechanisms. GCN2 kinase phosphorylates the eukaryotic initiation factor $2 \alpha$ (eIF $2 \alpha$ ), leading to the slower formation of the ternary complex, which is required for initiation of translation. However, mRNAs with many upstream open reading frames (uORFs) are preferentially translated, since slower ternary complex formation allows the small ribosomal subunit to bypass the intermediate uORFs and reach the protein coding sequence $(8,9)$. mTORC1 inhibition downregulates global protein translation by preventing the phosphorylation and activation of $70 \mathrm{kDa}$ ribosomal protein S6 kinase (p70S6K), as well as the phosphorylation and inhibition of and eIF4E binding protein 1 (4E-BP1). Unphosphorylated 4E-BP1 inhibits eIF4E required for cap-dependent mRNA translation. By 
acting in such a way, the inhibition of mTORC1 facilitates the cap-independent translation of mRNAs that contain internal ribosomal entry sites (IRES) (10,11).

Although GCN2 kinase and mTORC1 sense amino acid deprivation and affect protein translation through different mechanisms, both have been shown to suppress T-cell effector function. mTORC1 inhibitors are already components of immunosuppressive regiments in solid organ transplantation (12). Experimental data also indicate that GCN2 kinase activation may play a role in the prevention of allograft rejection. IDO exerts its immunosuppressive properties mainly through tryptophan depletion and GCN2 kinase activation $(7,13,14)$. The immunosuppressive role of IDO has been confirmed in animal models of allotransplantation (15-17). Importantly, the role of IDO in the downregulation of the adaptive immune response was revealed for the first time in an animal study which demonstrated that IDO expression in paternally-derived placental trophoblasts contributes to a successful semi-allogenic pregnancy (18).

In this study, the effects of GCN2 kinase activation or mTORC1 inhibition on primary human alloreactive CD4 ${ }^{+} \mathrm{T}$-cells were compared under the same experimental conditions. The two-way mixed lymphocyte reaction (MLR), as a model of alloreactivity (19), the GCN2 kinase activator, tryptophanol (TRP) $(14,20)$, and the mTORC1 inhibitor, rapamycin (RAP), were used (10). The concentration of TRP was selected to inhibit T-cell proliferation with negligible toxicity according to previous studies $(7,13,14)$. The concentration of RAP was selected to be within the recommended range of blood levels of kidney transplant recipients (12).

The results confirmed that in primary human alloreactive $\mathrm{CD} 4^{+} \mathrm{T}$-cells, the two systems that sense amino acid deprivation affect cell proliferation, apoptosis and differentiation in different ways or through different mechanisms.

\section{Materials and methods}

Subjects. Blood samples were collected from 8 unrelated healthy volunteers ( 5 females and 3 males, 40.8 \pm 7.2 years old). An informed consent was obtained from each individual prior to enrollment and the Ethics Committee of the Medical School of the University of Thessaly, Larissa, Greece gave its approval for the study protocol.

Peripheral blood mononuclear cell (PBMC) isolation and culture. PBMCs were isolated from whole blood by FicollHypaque density gradient centrifugation (Histopaque 1077; Sigma-Aldrich, St. Louis, MO, USA) and counted under an optical microscopy on a Neubauer plaque. Cell viability was assessed by trypan blue assay (Sigma-Aldrich).

The PBMCs were resuspended in RPMI-1640 medium with L-glutamine and $10 \mathrm{mM}$ 4-(2-hydroxyethyl)-1-piperazineethanesulfonic acid (HEPES) supplemented with $10 \%$ fetal bovine serum and antibiotic-antimycotic solution (both from Sigma-Aldrich). Isolated PBMCs from the enrolled subjects were coupled in order to set up 8 different MLRs. All cultures were performed at $37^{\circ} \mathrm{C}$ in a humidified atmosphere containing $5 \% \mathrm{CO}_{2}$.

Cytotoxicity of the evaluated compounds and cell proliferation in MLRs. MLRs were performed in 96-well plates for 7 days in the presence or not of $0.25 \mathrm{mM}$ TRP (Sigma-Aldrich) or 10 nM RAP (Tebu-bio, Le Perray-en-Yvelines Cedex, France). The number of PBMCs from each member of the MLR couple was $5 \times 10^{4}$, summing it up to $1 \times 10^{5} \mathrm{PBMCs}$ in total in each well.

At the end of the 7-day period, the cytotoxicity of TRP or RAP was assessed by lactate dehydrogenase (LDH) release assay using the Cytotox Non-Radioactive Cytotoxic assay kit (Promega Corp., Madison, WI, USA) according to the protocol provided by the manufacturer. Cytotoxicity was calculated using the following equation: cytotoxicity $(\%)=(\mathrm{LDH}$ in the supernatant/total LDH) x100. A total of 8 MLRs were performed in triplicate with the results for each MLR referring to the mean of the 3 measurements.

At the end of the 7-day period, cell proliferation was assessed by Cell Proliferation enzyme-linked immunosorbent assay (ELISA) (Roche Diagnostics, Indianapolis, IN, USA) using bromodeoxyuridine (BrdU) labeling and immunoenzymatic detection according to the manufacturer's instructions. Cultures of $1 \times 10^{5}$ resting PMBCs per well were used as the controls. The proliferation index (PI) was calculated as the ratio of the optical density (OD) derived from each MLR to the mean of the ODs derived from the control resting PBMCs of the 2 members of each MLR pair. A total of 8 MLRs were performed in triplicate with the results for each MLR referring to the mean of the 3 measurements.

Assessment of GCN2 kinase, mTORC1 activity, signature transcription factors of $\mathrm{CD}^{+} T$-cell subsets and key proteins involved in the proliferation, apoptosis and differentiation of $C D 4^{+} T$-cells isolated from the MLRs. A total of 8 MLRs were performed in 12-well plates for 7 days in the presence or not of $0.25 \mathrm{mM}$ TRP or $10 \mathrm{nM}$ RAP. The number of PBMCs for each member of the MLR couple was $5 \times 10^{5}$, summing up to $1 \times 10^{6}$ PBMCs in each well. At the end of the 7-day period, $\mathrm{CD} 4^{+} \mathrm{T}$-cells were isolated from the MLRs by negative selection using the $\mathrm{CD}^{+} \mathrm{T}$ cell isolation kit, human (Miltenyi Biotec $\mathrm{GmbH}$, Bergisch Gladbach, Germany).

The isolated $\mathrm{CD}^{+}$T-cells were counted via optical microscopy on a Neubauer plaque and cell viability was determined by trypan blue assay. PBMCs were isolated from whole blood by FicollHypaque density gradient centrifugation (Histopaque 1077; Sigma-Aldrich) and counted via optical microscopy using an optical microscope (Axiovert $40 \mathrm{C}$; Carl Zeiss AG, Oberkochen, Germany) and a Neubauer chamber (Paul Marienfeld GmbH, Lauda-Königshofen, Germany). Cell viability was assessed by trypan blue staining (Sigma-Aldrich) recommended for use in dye exclusion procedures for viable cell counting, based on the principle that live (viable) cells do not take up trypan blue, whereas dead (non-viable) cells do.

For western blot analysis, equal numbers of T-cells from each MLR were lysed using the T-PER tissue protein extraction reagent (Thermo Fisher Scientific, Rockford, IL, USA) supplemented with protease and phosphatase inhibitors (Sigma-Aldrich and Roche Diagnostics). Protein was quantified by Bradford assay (Sigma-Aldrich) and $10 \mu \mathrm{g}$ from each sample were electrophorized on a sodium dodecyl sulfate (SDS) polyacrylamide gel (Invitrogen, Life Technologies, Carlsbad, CA, USA). Subsequently, the proteins were transferred onto polyvinylidene difluoride (PVDF) membranes (Invitrogen, Life Technologies). The blots were incubated with the primary antibody for $16 \mathrm{~h}$, 
followed by the secondary antibody (anti-rabbit IgG, HRP-linked antibody; cat. no. 7074; Cell Signaling Technology, Danvers, MA, USA) for $30 \mathrm{~min}$. A benchmark pre-stained protein ladder (Invitrogen, Life Technologies) was used as a marker. Bands were visualized by enhanced chemiluminescent detection using the LumiSensor Plus Chemiluminescent HRP Substrate kit (GenScript, Piscataway, NJ, USA). In the case of reprobing the PVDF blots, the previous primary and secondary antibody were removed using Restore Western Blot Stripping Buffer (Thermo Fisher Scientific) according to the manufacturer's instructions. Analysis was performed using the ImageJ software (National Institute of Health, Bethesda, MD, USA).

The primary antibodies used for western blot analysis were specific for the substrate of GCN2 kinase eukaryotic initiation factor $2 \alpha$ phosphorylated at serine 51 (p-eIF2 $\alpha$; cat. no. 9721) and the substrate of mTORC1 p70S6 kinase phosphorylated at threonine 389 (p-p70S6K; cat. no. 9234) (both from Cell Signaling Technology, Danvers, MA, USA). In addition, specific antibodies against the Th1, Th2, Th17 and Treg signature transcription factors, T-box transcription factor TBX21 (T-bet; cat. no. 13232), trans-acting T-cell-specific transcription factor GATA-3 (GATA-3; cat. no. 5852) (both from Cell Signaling Technology), retinoic acid receptor related orphan receptor $\gamma \mathrm{t}$ (ROR $\gamma \mathrm{t}$; cat. no. orb6888) (Biorbyt, Cambridge, UK) and Forkhead box P3 (FoxP3; cat. no. 5298) (Cell Signaling Technology), respectively were used. Finally, specific antibodies were used for the detection of the tumor suppressor p53 (cat. no. 9282), cyclin dependent kinase inhibitor p21WAF1 (p21; cat. no. 2947), cleaved caspase-3 at Asp175 (CC3; cat. no. 9664) (all from Cell Signaling Technology), hypoxia-inducible factor-1 $\alpha$ (HIF-1 $\alpha$ ) (cat. no. sc-10790; Santa Cruz Biotechnology, Dallas, TX, USA) and LDH-A (cat. no. 2012; Cell Signaling Technology). All the results of western blot analysis were normalized to $\beta$-actin (cat. no. 4967; Cell Signaling Technology).

Assessment of interferon- $\gamma(I F N-\gamma)$, interleukin (IL)-4, IL-17 and IL-10 production in MLRs. A total of 8 MLRs were performed in 12-well plates in the presence or not of $0.25 \mathrm{mM}$ TRP or $10 \mathrm{nM}$ RAP, with the cell number of each PBMC population in the MLR context remaining the same as before. After 7 days, supernatants from each MLR were collected and IFN- $\gamma$, IL-4, IL-17 and IL-10 were measured by means of ELISA.

All ELISA kits for the measurement of cytokine production were purchased from R\&D Systems (Minneapolis, MN, USA). The sensitivity of the human IFN- $\gamma$ quantikine ELISA kit is $<8 \mathrm{pg} / \mathrm{ml}$, that of the human IL-4 quantikine ELISA kit $<10 \mathrm{pg} / \mathrm{ml}$, that of the human IL-17 quantikine ELISA kit $<15 \mathrm{pg} / \mathrm{ml}$ and that of the human IL-10 quantikine ELISA kit $<3.9 \mathrm{pg} / \mathrm{ml}$.

Statistical analysis. The normality of the evaluated variables was assessed and confirmed by a one-sample KolmogorovSmirnov test. For comparisons of the means, a paired-sample $\mathrm{t}$-test was used. The results are expressed as the means \pm standard deviation and a value of $\mathrm{p}<0.05$ was considered to indicate a statistically significant difference.

In case of the western blot analysis, since the original results were expressed as optical densities (OD), p-values were calculated by comparing the means of OD. Statistical analysis after normalization to the control OD values was avoided for

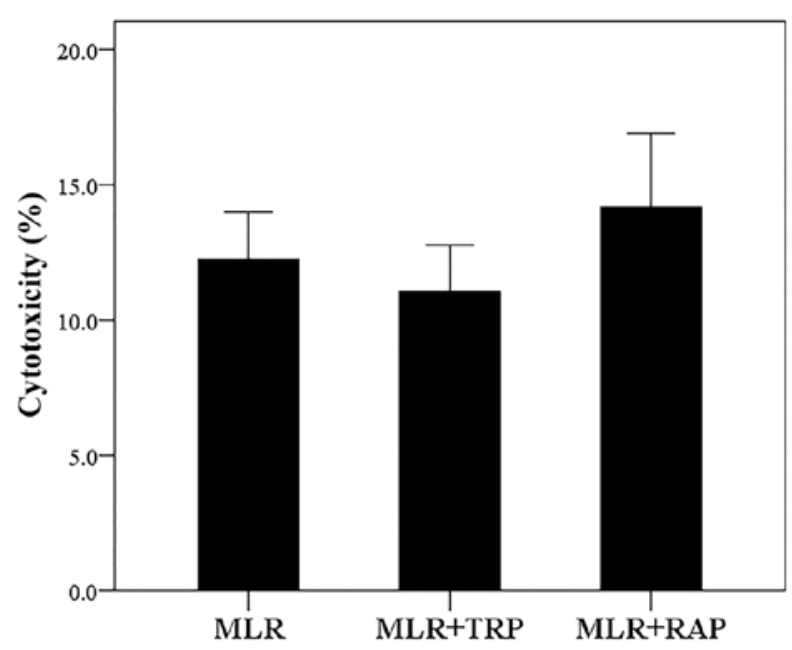

Figure 1. Cytotoxicity of tryptophanol (TRP) or rapamycin (RAP) in mixed lymphocyte reactions (MLRs). A total of 8 different MLRs were performed in triplicate and in the presence or not of $0.25 \mathrm{mM}$ TRP or $10 \mathrm{nM}$ RAP. Neither TRP nor RAP exhibited considerable toxicity. Error bars correspond to the standard deviation.

preventing the violation of the prerequisite for normal distribution of the compared variables when applying parametric statistical tests. However, for the reader's convenience, in the text, the results are expressed and depicted following normalization of values to the control group.

\section{Results}

In MLRs, both TRP and RAP exhibit negligible cytotoxicity. Compared to the untreated MLRs, neither treatment with TRP at a concentration of $0.25 \mathrm{mM}$ nor treatment with RAP at a concentration of $10 \mathrm{nM}$ resulted in considerable cytotoxicity. The cytotoxicity of MLR, MLR +TRP and MLR +RAP was $12.25 \pm 1.75,11.06 \pm 1.72$ and $14.19 \pm 2.71 \%$, respectively (Fig. 1).

In $\mathrm{CD}^{+}{ }^{+}$-cells, TRP activates GCN2 kinase, whereas RAP inhibits mTORCl. In the $\mathrm{CD}^{+} \mathrm{T}$-cells isolated from the MLRs treated with TRP, the level of phosphorylation of the GCN2 kinase substrate, eIF2 $\alpha$, was increased to a factor of $3.07 \pm 1.40(p<0.001)$. Treatment of the MLRs with RAP did not alter the p-eIF $2 \alpha$ level significantly. More precisely, in this case, the level of p-eIF $2 \alpha$ was altered to a factor of $1.25 \pm 0.82$ ( $\mathrm{p}=0.732$; Fig. 2 ).

Treatment of the MLRs with RAP resulted in the decreased phosphorylation of the mTORC1 substrate, p70S6K, to a factor of $0.59 \pm 0.09(p<0.001)$ in the $\mathrm{CD}^{+} \mathrm{T}$-cells. Treatment of the MLRs with TRP did not affect the p-p70S6K level, only altering it to a factor of $0.99 \pm 0.10$ ( $p=0.668$; Fig. 2).

In MLRs, both TRP and RAP decrease cell proliferation. In the untreated MLRs, the PI was 4.42 \pm 0.46 . Treatment with TRP decreased the PI to $2.28 \pm 0.30(\mathrm{p}<0.001)$. RAP also decreased PI significantly to $1.56 \pm 0.39$ ( $<<0.001$; Fig. 3$)$.

In $C D 4^{+} T$-cells, TRP increases $p 53, p 21$ and CC3 expression, whereas RAP decreases p53 and p21 and increases 

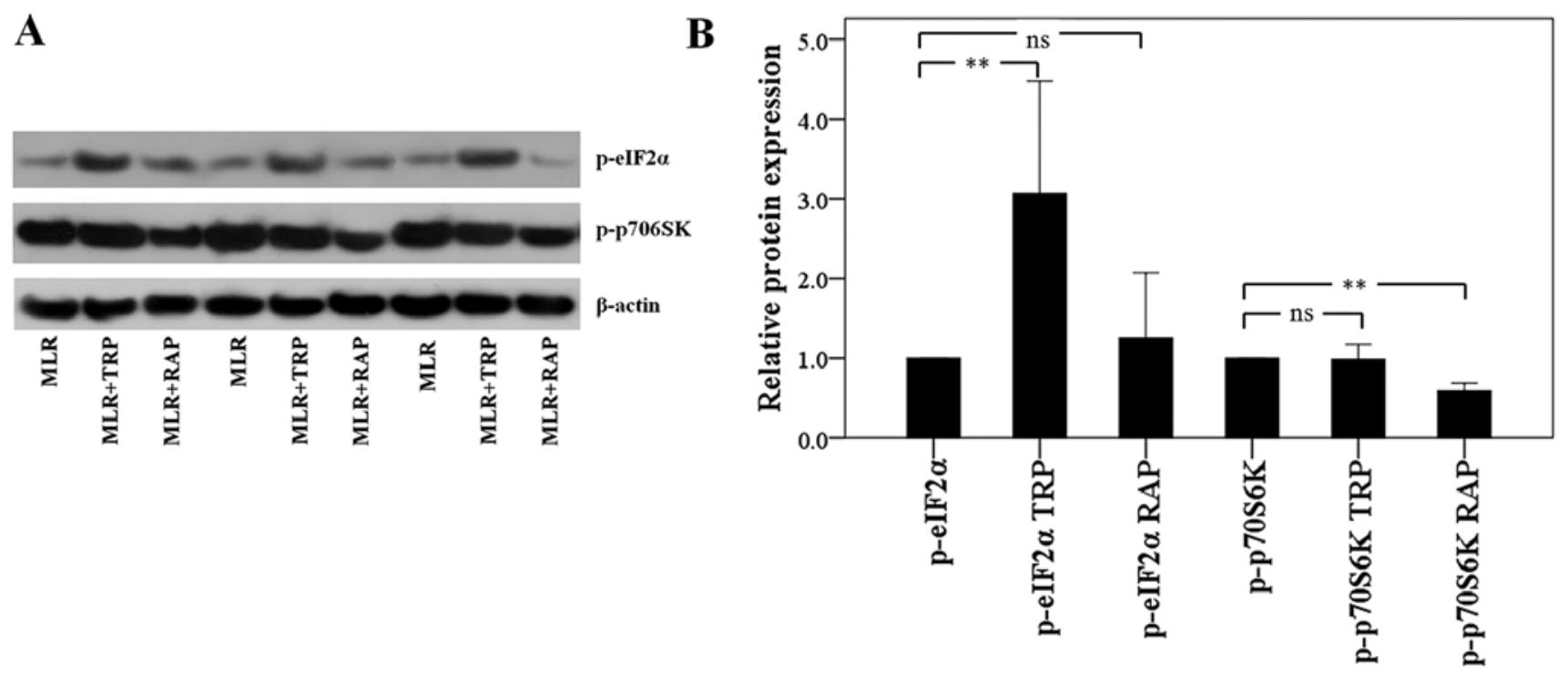

Figure 2. Effect of tryptophanol (TRP) and rapamycin (RAP) on general control nonderepressible 2 (GCN2) kinase and mammalian target of rapamycin complex 1 (mTORC1) activities in $\mathrm{CD}^{+}{ }^{+} \mathrm{T}$-cells isolated from the mixed lymphocyte reactions (MLRs). A total of 8 different MLRs were performed and the $\mathrm{CD} 4^{+} \mathrm{T}$-cells were then isolated, proteins extracted and analyzed by western blot analysis. (A) The results of 3 experiments are depicted. (B) TRP activated the GCN2 kinase since it enhanced the phosphorylation of its substrate eukaryotic initiation factor $2 \alpha$ (eIF2 $\alpha$ ), whereas it did not affect the phosphorylation of the mTORC1 substrate p70S6K. RAP inhibited the mTOR since it decreased the phosphorylation of its substrate p70S6K, while it did not affect the phosphorylation of the GCN2 kinase substrate eIF2 $\alpha$. Error bars correspond to the standard deviation. ns, not significant $(\mathrm{p} \geq 0.05) ;{ }^{* *} \mathrm{p}<0.001$.

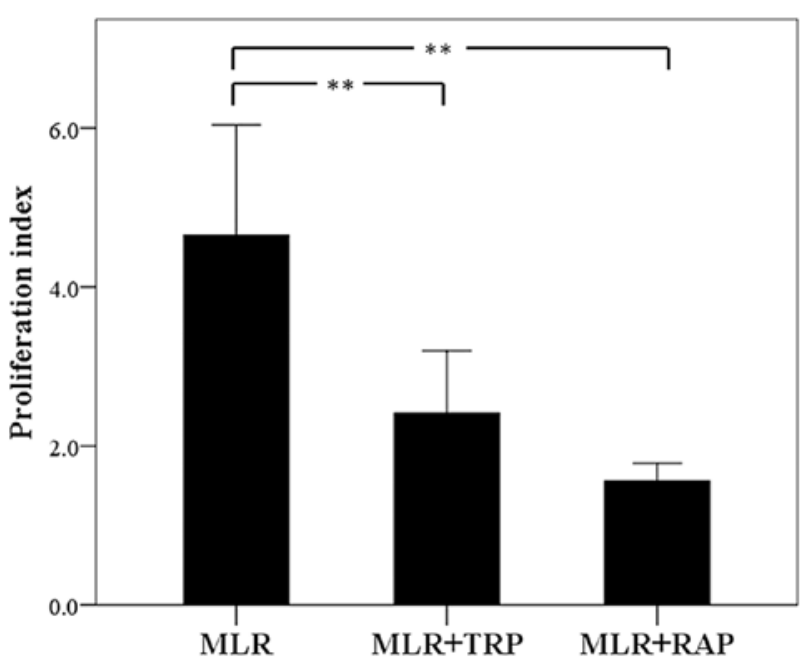

Figure 3. Effect of tryptophanol (TRP) or rapamycin (RAP) on cell proliferation in mixed lymphocyte reactions (MLRs). A total of 8 different MLRs were performed in triplicate. Both TRP and RAP significantly decreased cell proliferation. Error bars correspond to the standard deviation, ${ }^{* *} \mathrm{p}<0.001$.

CC3 expression. In the CD4 ${ }^{+} \mathrm{T}$-cells isolated from the MLRs treated with TRP, p53 expression was increased to a factor of $1.50 \pm 0.33(\mathrm{p}<0.001)$. The expression of the $\mathrm{p} 53$ transcriptional target, p21, was also increased to a factor of $1.40 \pm 0.10(\mathrm{p}<0.001)$. Finally, the level of CC3 was also found to be increased to a factor of 3.02 \pm 2.21 ( $<<0.001$; Fig. 4).

Although treatment of the MLRs with RAP also resulted in an increase in $\mathrm{CC} 3$ expression in the $\mathrm{CD}^{+}{ }^{+} \mathrm{T}$-cells to a factor of $3.35 \pm 2.16(p<0.001)$, the effects of treatment with RAP on the p53 and p21 levels were the opposite to those observed following treatment with TRP. More precisely, RAP decreased both the p53 and p21 expression levels to a factor of $0.56 \pm 0.19(\mathrm{p}=0.004)$ and $0.69 \pm 0.13(\mathrm{p}=0.001)$, respectively (Fig. 4).

In $\mathrm{CD}^{+}$T-cells, TRP and RAP decrease GATA-3 expression, but RAP also decreases T-bet and ROR $\mathrm{T}$ t expression, and increases FoxP3 expression. Treatment of the MLRs with TRP affected only GATA-3 expression in the CD4 ${ }^{+}$T-cells. The level of this Th2 signature transcription factor decreased to a factor of $0.55 \pm 0.19(\mathrm{p}<0.001)$. On the contrary, TRP did not affect the expression of the Th1 cell signature transcription factor, T-bet, that of the Th17 cell signature transcription factor, ROR $\gamma$ t, or that of the Treg cell signature transcription factor, FoxP3. The levels of these factors were only altered to a factor of $0.99 \pm 0.12(\mathrm{p}=0.722), 0.96 \pm 0.17(\mathrm{p}=0.589)$ and $1.05 \pm 0.19$ ( $\mathrm{p}=0.568)$, respectively (Fig. 5).

In contrast to TRP, treatment of the MLRs with RAP altered the expression levels of all the evaluated CD4 ${ }^{+} \mathrm{T}$-cell subset signature transcription factors. Treatment with RAP resulted in the decreased expression of T-bet, GATA-3 and ROR $\gamma t$ to a factor of $0.47 \pm 0.09(\mathrm{p}<0.001), 0.47 \pm 0.11(\mathrm{p}<0.001)$ and $0.41 \pm 0.19(\mathrm{p}=0.001)$, respectively. In the same cellular context, the presence of RAP significantly upregulated FoxP3 to a factor of $1.97 \pm 1.15$ ( $\mathrm{p}=0.003$; Fig. 5 ).

In $\mathrm{CD}^{+} \mathrm{T}$-cells, TRP increases HIF-1 $\alpha$ expression and RAP decreases HIF-1 a expression, but both result in the decreased expression of $L D H-A$. Treatment of the MLRs with TRP increased the expression of HIF-1 $\alpha$, which enhances the ratio of Th17/Treg differentiation, in $\mathrm{CD}^{+}{ }^{+} \mathrm{T}$-cells to a factor of $1.44 \pm 0.18(\mathrm{p}<0.001)$. However, the level of its transcriptional target, LDH-A, was found decreased to a factor of $0.56 \pm 0.08$ (p<0.001; Fig. 6). 

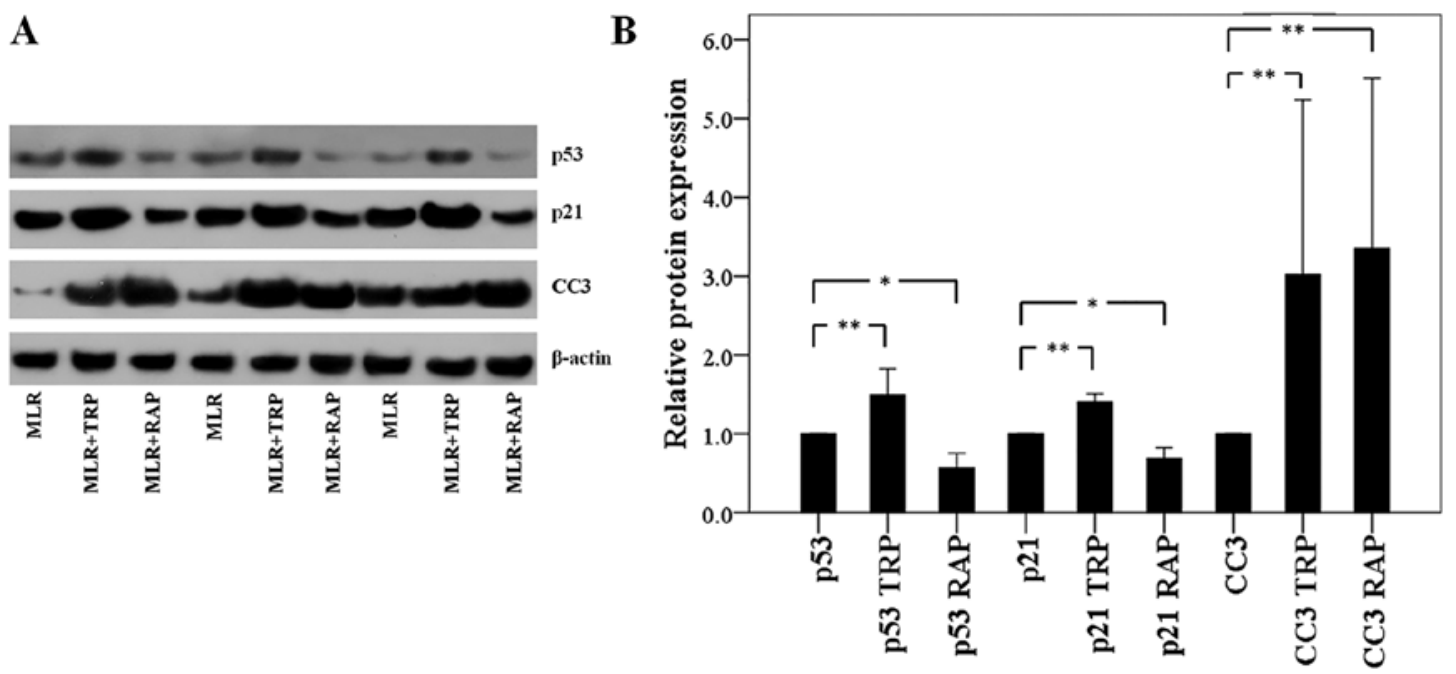

Figure 4. Effect of tryptophanol (TRP) and rapamycin (RAP) on p53, p21 and CC3 expression in CD4 ${ }^{+}$T-cells isolated from the mixed lymphocyte reactions (MLRs). A total of 8 different MLRs were performed and the $\mathrm{CD}^{+}$T-cells were then isolated, proteins extracted and analyzed by western blot analysis (A) The results of 3 experiments are depicted in. (B) TRP increased p53 expression, that of its transcriptional target and cell cycle regulator p21 and that of the activated pro-apoptotic caspase, CC 3 . RAP also increased CC3, but decreased p53 and p21 expression. Error bars correspond to the standard deviation, ${ }^{*}$ p $<0.05$ and ${ }^{* *} \mathrm{p}<0.001$.

A



B

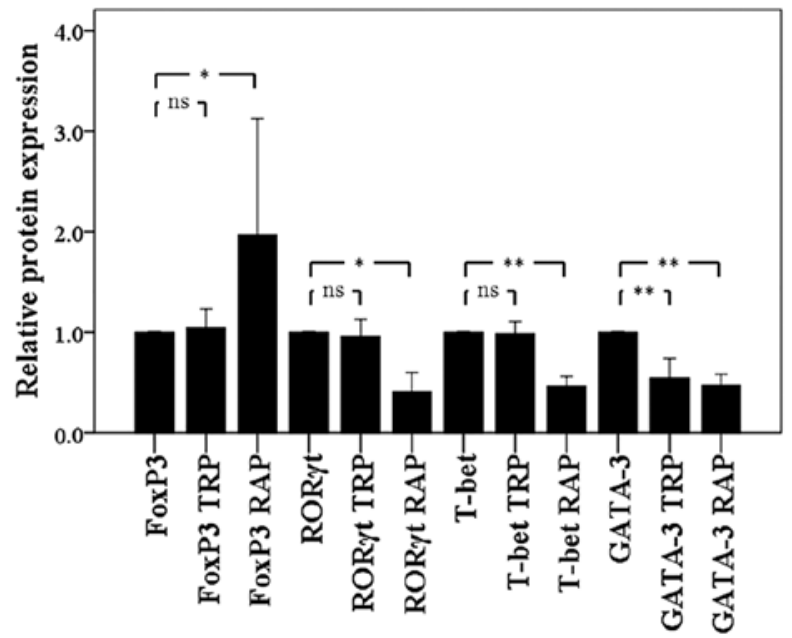

Figure 5. Effect of tryptophanol (TRP) and rapamycin (RAP) on the expression of CD4 ${ }^{+}$T-cell subset signature transcription factors. A total of 8 different mixed lymphocyte reactions (MLRs) were performed and the $\mathrm{CD} 4^{+} \mathrm{T}$-cells were then isolated, proteins extracted and analyzed by western blot analysis. (A) The results of 3 experiments are depicted. (B) From the evaluated transcription factors, TRP only decreased the expression of the Th2 signature transcription factor, GATA-3. RAP affected all the evaluated transcription factors. Similar to TRP, RAP also decreased GATA-3 expression, but also that of the Th1 and Th17 signature transcription factors, T-bet and receptor related orphan receptor $\gamma \mathrm{t}(\mathrm{ROR} \gamma \mathrm{t})$. On the contrary, RAP increased the expression of the Treg signature transcription factor, Forkhead box P3 (FoxP3). Error bars correspond to the standard deviation. ns, not significant ( $\mathrm{p} \geq 0.05$ ); ${ }^{*} \mathrm{p}<0.05$ and ${ }^{* *} \mathrm{p}<0.001$.

Treatment with RAP decreased both the HIF-1 $\alpha$ and LDH-A levels to a factor of $0.66 \pm 0.15(\mathrm{p}=0.001)$ and $0.49 \pm 0.20$ ( $\mathrm{p}=0.001)$, respectively (Fig. 6).

In MLRs, TRP and RAP decrease IL-4 expression and increase IL-10 expression, but RAP also decreases IFN- $\gamma$ and $I L-17$ expression. The concentration of the Th1 cell signature cytokine, IFN- $\gamma$, was not altered significantly in the supernatants of the TRP-treated MLRs. The level of IFN $-\gamma$ was $121.50 \pm 15.98 \mathrm{pg} / \mathrm{ml}$ in the untreated MLRs and $109.00 \pm 19.40 \mathrm{pg} / \mathrm{ml}$ in the TRP-treated MLRs $(\mathrm{p}=0.205)$. On the contrary, treatment with RAP decreased IFN- $\gamma$ expression to $44.25 \pm 11.68 \mathrm{pg} / \mathrm{ml}$ (p<0.001; Fig. 7A).
Treatment of MLRs with TRP decreased the production of the Th2 cell signature cytokine, IL-4, from $110.12 \pm 20.79$ to $71.00 \pm 15.22 \mathrm{pg} / \mathrm{ml}(\mathrm{p}<0.001)$. Treatment with RAP also decreased the IL- 4 concentration to $37.50 \pm 13.38 \mathrm{pg} /$ $\mathrm{ml}(\mathrm{p}<0.001$; Fig. 7B).

The expression of the signature cytokine of the Th17 cells, IL-17, remained unaffected by TRP, with a concentration of $225.37 \pm 27.71 \mathrm{pg} / \mathrm{ml}$ in the untreated MLRs and $217.12 \pm 21.14 \mathrm{pg} / \mathrm{ml}$ in the TRP-treated MLRs ( $\mathrm{p}=0.382$ ). However, treatment with RAP significantly decreased the IL-17 concentration to $67.25 \pm 20.71 \mathrm{pg} / \mathrm{ml}$ ( $\mathrm{p}<0.001$; Fig. 7C).

As regards the cytokine, IL-10, which is produced by Treg and by certain macrophages, treatment of the MLRs 

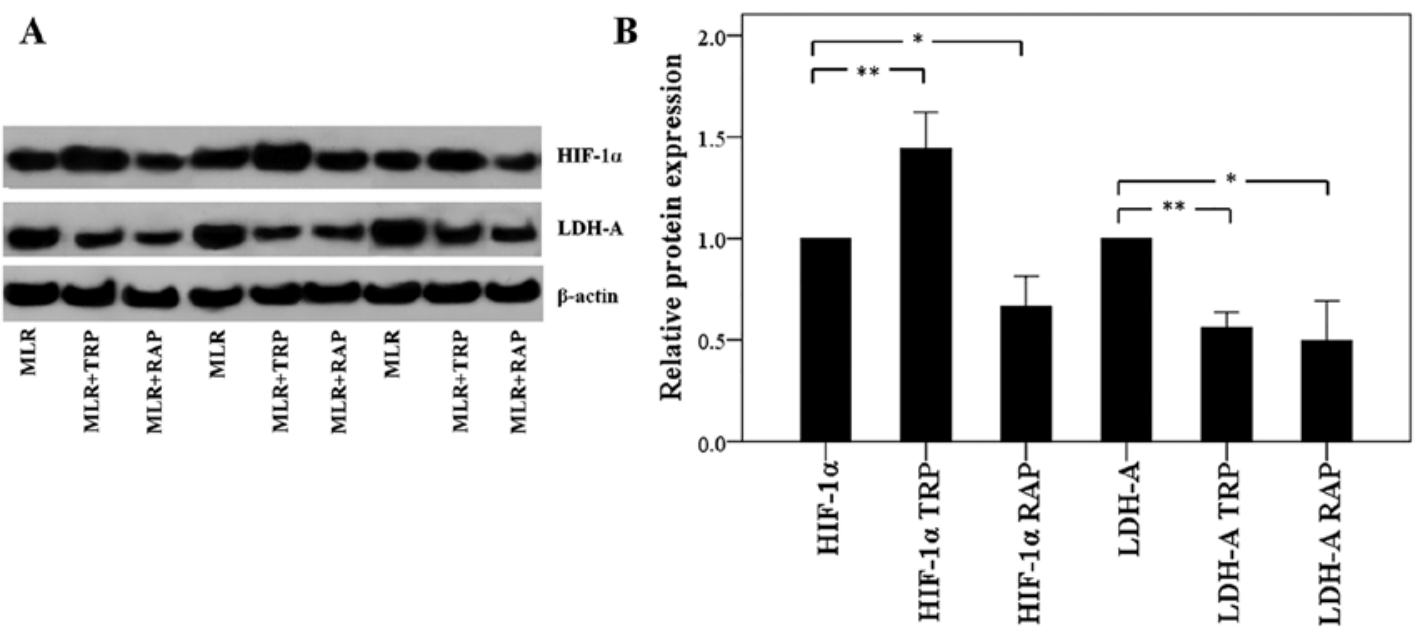

Figure 6. Effect of tryptophanol (TRP) and rapamycin (RAP) on HIF-1 $\alpha$ and its activity in CD4+ T-cells isolated from the mixed lymphocyte reactions (MLRs). A total of 8 different MLRs were performed and the CD4 ${ }^{+}$T-cells were then isolated, proteins extracted and analyzed by western blot analysis. (A) The results of 3 experiments are depicted. (B) Expression of HIF-1 $\alpha$, which enhances the Th17/Treg ratio, was differentially regulated by TRP and RAP. TRP enhanced the HIF-1 $\alpha$ level, whereas RAP decreased it. As regards HIF-1 $\alpha$ transcriptional activity, assessed by the expression of its transcriptional target, lactate dehydrogenase-A (LDH-A), both TRP and RAP decreased it. Error bars correspond to the standard deviation, ${ }^{*}<<0.05$ and ${ }^{* *} \mathrm{p}<0.001$.
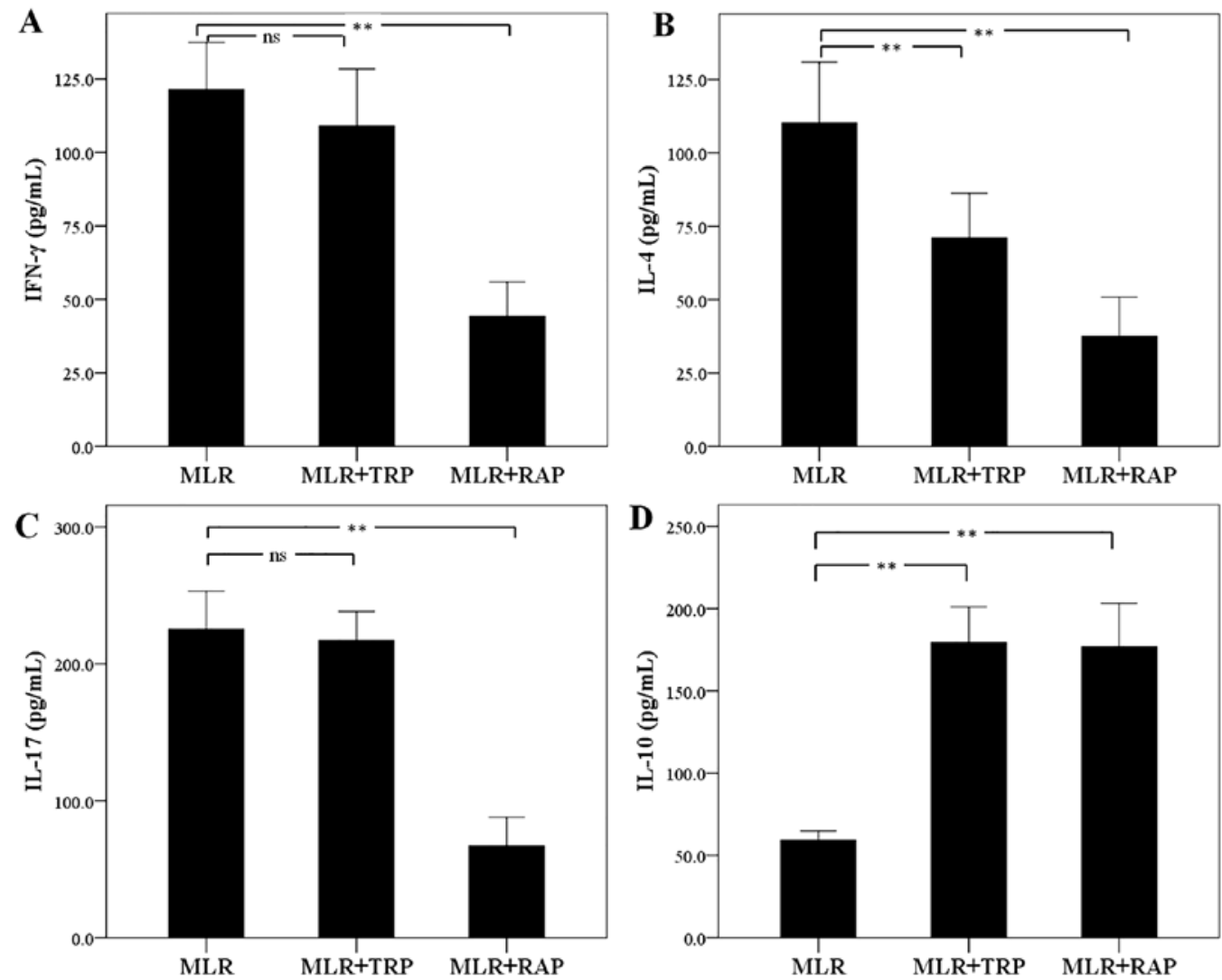

Figure 7. Effect of tryptophanol (TRP) and rapamycin (RAP) on the production of interferon- $\gamma$ (IFN- $\gamma$ ), interleukin (IL)-4, IL-17 and IL-10 in mixed lymphocyte reactions (MLRs). A total of 8 different MLRs were performed and the supernatants were then collected and cytokines were measured by enzyme-linked immunosorbent assay (ELISA). (A) A major source of IFN- $\gamma$ is the Th1 subset. RAP decreased its concentration, whereas TRP had no effect. (B) IL-4 is produced mainly by Th2 cells and both TRP and RAP decreased its concentration. (C) The Th17 signature cytokine IL-17 remained unaffected by TRP, but RAP decreased its expression significantly. (D) Finally, the expression of IL-10, which is produced by both Tregs and certain macrophages, increased in MLRs treated with TRP or RAP. Error bars correspond to the standard deviation. ns, not significant $(\mathrm{p} \geq 0.05) ;{ }^{* *} \mathrm{p}<0.001$.

with both TRP and RAP increased its concentration. More precisely, TRP increased the IL-10 concentration from $59.26 \pm 5.55$ in the untreated cells to $179.37 \pm 21.72 \mathrm{pg} /$ $\mathrm{ml}(\mathrm{p}<0.001)$ in the TRP-treated cells. Treatment with RAP increased the IL-10 concentration to $176.75 \pm 26.48 \mathrm{pg} / \mathrm{ml}$ $(\mathrm{p}<0.001$; Fig. 7D). 


\section{Discussion}

In this study, the effects of GCN2 kinase activation or mTORC1 inhibition, the two systems able to sense amino acid deprivation, on primary human alloreactive $\mathrm{CD} 4{ }^{+} \mathrm{T}$-cells were evaluated. For this purpose, the GCN2 kinase activator, TRP, and the mTORC1 inhibitor, RAP, were used at concentrations selected according to previous experimental data or clinical recommendations $(7,12-14)$. Cytotoxicity assay revealed that neither $0.25 \mathrm{mM}$ TRP nor $10 \mathrm{nM}$ RAP exhibited considerable toxicity in the context of MLRs.

In order to determine whether the working concentrations of TRP and RAP used were adequate, their effects on GCN2 kinase and mTORC1 activities were evaluated in $\mathrm{CD}^{+}{ }^{+} \mathrm{T}$-cells isolated from the MLRs. TRP enhanced the activity of GCN2 kinase, assessed by the level of phosphorylation of its substrate, eIF2 $\alpha$ (9), whereas mTORC1 activity remained unaffected. Accordingly, RAP reduced mTORC1 activity, assessed by the level of phosphorylation of its substrate, p70S6K $(10,11)$, without altering GCN2 kinase activity.

In MLRs, both TRP and RAP decreased cell proliferation. They also induced the apoptosis of $\mathrm{CD} 4^{+} \mathrm{T}$-cells isolated from the MLRs, assessed by the level of activated CC3, a point at which all the apoptotic pathways converge (21). It is known that in eukaryotic cells, both GCN2 kinase activation and mTORC1 inhibition decrease cell proliferation and induce apoptosis. Halofuginone, which activates GCN2 kinase and also inhibits transforming growth factor- $\beta$ (TGF- $\beta$ ) signal transduction (22), suppresses T-cell proliferation and induces apoptosis (23). In addition, IDO through tryptophan depletion and GCN2 kinase activation, suppresses proliferation $(7,13)$, and induces the apoptosis of T-cells $(24,25)$. The inhibition of mTORC1 also decreases the proliferation and induces the apoptosis of T-cells $(10,26,27)$.

The tumor suppressor p53 plays a central role in controlling cell proliferation by inducing G1-phase cell cycle arrest through the activation of the transcription of $\mathrm{p} 21$. It also induces apoptosis (28). In the case of TRP treatment, decreased cell proliferation and increased $\mathrm{CC} 3$ expression were accompanied by an increased $\mathrm{p} 53$ expression in $\mathrm{CD} 4^{+} \mathrm{T}$-cells isolated from the MLRs. TRP also increased p21 expression. Thus, upon GCN2 kinase activation, p53 increases and may contribute to both decreased proliferation and increased apoptosis. Interestingly, a previous study demonstrated that IDO, through TRP depletion and GCN2 kinase activation, increases p53 expression in human T-cells, resulting in a p53-dependent suppression of cell proliferation (13). Contrary to TRP, RAP decreased 553 expression and the expression of its transcriptional target, $\mathrm{p} 21$, in $\mathrm{CD}_{4}{ }^{+} \mathrm{T}$-cells isolated from the MLRs, indicating that this compound decreases cell proliferation and induces apoptosis in a p53-independent manner, a result that also has been detected in various cancer cell lines $(29,30)$, the human Molt-4 T-cell line included (27).

Next, the effects of GCN2 kinase activation or mTOR inhibition on $\mathrm{CD}^{+} \mathrm{T}$-cell differentiation were evaluated by assessing the expression of the signature transcription factors of CD4 ${ }^{+}$T-cell subsets, FoxP3 for Treg, ROR $\gamma \mathrm{t}$ for Th17, T-bet for Th1 and GATA-3 for Th2 (31). TRP affected, and more precisely decreased, only the signature transcription factor of Th2, GATA-3. Generally, the effect of GCN2 kinase activation on $\mathrm{CD} 4^{+} \mathrm{T}$-cell differentiation is an area which has not been extensively studied. There are studies available on the effect of IDO on the differentiation of these cells, which however, concluded in contradictory results. For instance, it has been demonstrated that IDO promotes Treg differentiation (32), while others have failed to detect such an effect (33). The differential experimental systems and species used may be responsible for these discrepancies. In the present study, primary human cells and a validated model of alloreactivity were used. It is also notable that apart from GCN2 kinase activation, the IDO-produced kynurenine pathway products may play a role in $\mathrm{CD} 4^{+} \mathrm{T}$-cell differentiation (34). In this study, treatment of MLRs with RAP exerted a more profound effect on $\mathrm{CD}^{+} \mathrm{T}$-cell differentiation. RAP decreased the expression of the signature transcription factors of all the evaluated effector CD4 ${ }^{+}$T-cell subsets, i.e., T-bet of Th1, GATA-3 of Th2 and ROR $\gamma t$ of Th17. On the contrary, RAP increased the expression of FoxP3, the signature transcription factor of Treg. This is in accordance with the fact that mouse mTORdeficient $\mathrm{CD}^{+}{ }^{+} \mathrm{T}$-cells fail to differentiate into Th1, Th2 and Th17 subsets under proper conditions, while they preferably differentiate into the Treg subset (35). However, between the two mTOR complexes, RAP inhibits only mTORC1 (10), and there are elegant studies showing that it is the mTORC2 that governs Th2 differentiation (36-38). Nevertheless, in the present study, cells were treated with RAP for 1 week, and such prolonged treatment with this compound has been shown to inhibit mTORC2 assembly and function (39).

The differentiation towards Th17 or Treg is of particular interest, since the Th17/Treg balance plays a significant role in various autoimmune diseases (31), as well as in organ transplantation (40). The Th17/Treg ratio is governed, and upregulated, by the transcription factor, HIF-1 $\alpha(41,42)$. In this study, treatment of MLRs with RAP resulted in a decreased HIF-1 $\alpha$ level in the $\mathrm{CD}^{+} \mathrm{T}$-cells, which was expected (43), and is in agreement with the RAP-induced increase in FoxP3 expression and a decrease in ROR $\gamma t$ expression. On the contrary, $\mathrm{CD} 4^{+} \mathrm{T}$-cells from MLRs treated with TRP exhibited higher HIF-1 $\alpha$ levels; however, the levels of FoxP3 or ROR $\gamma \mathrm{t}$ were not altered in these cells. The reason for GCN2 kinase-induced HIF-1 $\alpha$ upregulation remains to be evaluated, although it may be p53-mediated $(44,45)$. However, accumulated HIF-1 $\alpha$ is not always active (46). The discrepancy between the TRP-induced increase in HIF-1 $\alpha$ levels accompanied however by stable FoxP3 and ROR $\gamma$ t levels was evaluated by examining HIF- $1 \alpha$ activity on specific target proteins. Indeed, the expression of LDH-A, a well-known transcriptional target of HIF-1 $\alpha$, was assessed and found to be decreased. Consequently, accumulated HIF- $1 \alpha$ in $\mathrm{CD}^{+}$T-cells from TRP-treated MLRs remained non-functional. This may be the result of the competition between p53 and HIF- $1 \alpha$ for limited amounts of the transcriptional co-activator, p300 (47).

Finally, we evaluated the levels of signature cytokines for the evaluated Th subsets, i.e., of IFN- $\gamma$ for Th1, IL-4 for Th2, IL-17 for Th17 and IL-10 for Treg (31). RAP decreased the production of IFN- $\gamma$, IL-4 and IL-17, whereas it increased the level of IL-10. These changes were parallel to the observed alterations of the Th subset signature transcription factors. TRP reduced the IL- 4 concentration, which is in the same consensus with the decreased GATA-3 expression. However, TRP increased IL-10 production, whereas it did not affect the 
FoxP3 level. This discrepancy deserves evaluation, but may be the result of IL-10 production by other cell types. For instance, apart from Treg, M2 type macrophages produce IL-10, and IDO promotes this type of macrophages (48). RAP exerts the opposite effect (49), excluding M2 macrophages as an alternative source of IL-10 in RAP-treated MLRs.

In conclusion, in primary human alloreactive $\mathrm{CD} 4^{+} \mathrm{T}$-cells, the two systems that sense amino acid deprivation affect cell proliferation, apoptosis and differentiation in different ways or through different mechanisms. Both mTOR inhibition and GCN2 kinase activation exert immunosuppressive effects, since they inhibit cell proliferation and induce apoptosis. As regards $\mathrm{CD}^{+}{ }^{+}$T-cell differentiation, mTOR inhibition exerts a more profound effect, since it suppresses differentiation into Th1, Th2 and Th17, while it induces Treg differentiation. On the contrary, activation of GCN2 kinase suppresses only Th2 differentiation. Thus, GCN2 kinase is a potential target for novel immunosuppressive medications with possibly a more profound effect on the treatment of Th2-mediated disorders.

\section{References}

1. Bronte $\mathrm{V}$ and Zanovello P: Regulation of immune responses by L-arginine metabolism. Nat Rev Immunol 5: 641-654, 2005.

2. Mellor AL and Munn DH: IDO expression by dendritic cells: Tolerance and tryptophan catabolism. Nat Rev Immunol 4: 762-774, 2004.

3. Dong J, Qiu H, Garcia-Barrio M, Anderson J and Hinnebusch AG: Uncharged tRNA activates GCN2 by displacing the protein kinase moiety from a bipartite tRNA-binding domain. Mol Cell 6: 269-279, 2000.

4. Gallinetti J, Harputlugil E and Mitchell JR: Amino acid sensing in dietary-restriction-mediated longevity: Roles of signal-transducing kinases GCN2 and TOR. Biochem J 449: 1-10, 2013.

5. Sancak Y, Peterson TR, Shaul YD, Lindquist RA, Thoreen CC, Bar-Peled L and Sabatini DM: The Rag GTPases bind raptor and mediate amino acid signaling to mTORC1. Science 320: 1496-1501, 2008.

6. Kim E, Goraksha-Hicks P, Li L, Neufeld TP and Guan KL: Regulation of TORC1 by Rag GTPases in nutrient response. Nat Cell Biol 10: 935-945, 2008.

7. Eleftheriadis T, Pissas G, Antoniadi G, Liakopoulos V and Stefanidis I: Indoleamine 2,3-dioxygenase depletes tryptophan, activates general control non-derepressible 2 kinase and downregulates key enzymes involved in fatty acid synthesis in primary human CD4 ${ }^{+} \mathrm{T}$ cells. Immunology 146: 292-300, 2015.

8. Kilberg MS, Shan J and Su N: ATF4-dependent transcription mediates signaling of amino acid limitation. Trends Endocrinol Metab 20: 436-443, 2009.

9. Castilho BA, Shanmugam R, Silva RC, Ramesh R, Himme BM and Sattlegger E: Keeping the eIF2 alpha kinase Gcn 2 in check. Biochim Biophys Acta 1843: 1948-1968, 2014.

10. Laplante M and Sabatini DM: mTOR signaling at a glance. J Cell Sci 122: 3589-3594, 2009.

11. Ma XM and Blenis J: Molecular mechanisms of mTOR-mediated translational control. Nat Rev Mol Cell Biol 10: 307-318, 2009.

12. Shihab F, Christians U, Smith L, Wellen JR and Kaplan B: Focus on mTOR inhibitors and tacrolimus in renal transplantation: Pharmacokinetics, exposure-response relationships, and clinical outcomes. Transpl Immunol 31: 22-32, 2014.

13. Eleftheriadis T, Pissas G, Antoniadi G, Spanoulis A, Liakopoulos V and Stefanidis I: Indoleamine 2,3-dioxygenase increases p53 levels in alloreactive human $\mathrm{T}$ cells, and both indoleamine 2,3-dioxygenase and p53 suppress glucose uptake, glycolysis and proliferation. Int Immunol 26: 673-684, 2014.

14. Munn DH, Sharma MD, Baban B, Harding HP, Zhang Y, Ron D and Mellor AL: GCN2 kinase in T cells mediates proliferative arrest and anergy induction in response to indoleamine 2,3-dioxygenase. Immunity 22: 633-642, 2005.

15. Alexander AM, Crawford M, Bertera S, Rudert WA, Takikawa O, Robbins PD and Trucco M: Indoleamine 2,3-dioxygenase expression in transplanted NOD Islets prolongs graft survival after adoptive transfer of diabetogenic splenocytes. Diabetes 51: 356-365, 2002.
16. Beutelspacher SC, Pillai R, Watson MP, Tan PH, Tsang J, McClure MO, George AJ and Larkin DF: Function of indoleamine 2,3-dioxygenase in corneal allograft rejection and prolongation of allograft survival by over-expression. Eur J Immunol 36: 690-700, 2006.

17. Li Y, Tredget EE, Ghaffari A, Lin X, Kilani RT and Ghahary A: Local expression of indoleamine 2,3-dioxygenase protects engraftment of xenogeneic skin substitute. J Invest Dermatol 126: 128-136, 2006.

18. Munn DH, Zhou M, Attwood JT, Bondarev I, Conway SJ, Marshall B, Brown C and Mellor AL: Prevention of allogeneic fetal rejection by tryptophan catabolism. Science 281: 1191-1193, 1998.

19. Sato T, Deiwick A, Raddatz G, Koyama K and Schlitt HJ: Interactions of allogeneic human mononuclear cells in the two-way mixed leucocyte culture (MLC): Influence of cell numbers, subpopulations and cyclosporin. Clin Exp Immunol 115: 301-308, 1999.

20. Lowe G and Tansley G: An investigation of the mechanism of activation of tryptophan by tryptophanyl-tRNA synthetase from beef pancreas. Eur J Biochem 138: 597-602, 1984.

21. Fadeel B and Orrenius S: Apoptosis: A basic biological phenomenon with wide-ranging implications in human disease. J Intern Med 258: 479-517, 2005.

22. Pines $M$ and Spector I: Halofuginone - the multifaceted molecule. Molecules 20: 573-594, 2015.

23. Chu TL, Guan Q, Nguan CY and Du C: Halofuginone suppresses $\mathrm{T}$ cell proliferation by blocking proline uptake and inducing cell apoptosis. Int Immunopharmacol 16: 414-423, 2013.

24. Forouzandeh F, Jalili RB, Germain M, Duronio V and Ghahary A: Skin cells, but not $\mathrm{T}$ cells, are resistant to indoleamine 2, 3-dioxygenase IDO) expressed by allogeneic fibroblasts. Wound Repair Regen 16: 379-387, 2008.

25. Habibi D, Jalili RB, Forouzandeh F, Ong CJ and Ghahary A: High expression of IMPACT protein promotes resistance to indoleamine 2,3-dioxygenase-induced cell death. J Cell Physiol 225: 196-205, 2010.

26. Weichhart $\mathrm{T}$, Costantino $\mathrm{G}$, Poglitsch M, Rosner $\mathrm{M}$ Zeyda M, Stuhlmeier KM, Kolbe T, Stulnig TM, Hörl WH, Hengstschläger M, et al: The TSC-mTOR signaling pathway regulates the innate inflammatory response. Immunity 29 : 565-577, 2008.

27. Choi SJ, You HS and Chung SY: Rapamycin-induced cytotoxic signal transduction pathway. Transplant Proc 40: 2737-2739, 2008.

28. Brady CA and Attardi LD: p53 at a glance. J Cell Sci 123: 2527-2532, 2010.

29. Metcalfe SM, Canman CE, Milner J, Morris RE, Goldman S and Kastan MB: Rapamycin and p53 act on different pathways to induce G1 arrest in mammalian cells. Oncogene 15: 1635-1642, 1997.

30. Miyake N, Chikumi H, Takata M, Nakamoto M, Igishi T and Shimizu E: Rapamycin induces p53-independent apoptosis through the mitochondrial pathway in non-small cell lung cancer cells. Oncol Rep 28: 848-854, 2012.

31. Raphael I, Nalawade S, Eagar TN and Forsthuber TG: T cell subsets and their signature cytokines in autoimmune and inflammatory diseases. Cytokine 74: 5-17, 2015.

32. Fallarino F, Grohmann U, You S, McGrath BC, Cavener DR, Vacca C, Orabona C, Bianchi R, Belladonna ML, Volpi C, et al: The combined effects of tryptophan starvation and tryptophan catabolites down-regulate $T$ cell receptor zeta-chain and induce a regulatory phenotype in naive T cells. J Immunol 176: 6752-6761, 2006.

33. Ito H, Ando T, Ando K, Ishikawa T, Saito K, Moriwaki H and Seishima M: Induction of hepatitis B virus surface antigen-specific cytotoxic $\mathrm{T}$ lymphocytes can be up-regulated by the inhibition of indoleamine 2, 3-dioxygenase activity. Immunology 142: 614-623, 2014.

34. Mezrich JD, Fechner JH, Zhang X, Johnson BP, Burlingham WJ and Bradfield CA: An interaction between kynurenine and the aryl hydrocarbon receptor can generate regulatory $\mathrm{T}$ cells. J Immunol 185: 3190-3198, 2010.

35. Delgoffe GM, Kole TP, Zheng Y, Zarek PE, Matthews KL, Xiao B, Worley PF, Kozma SC and Powell JD: The mTOR kinase differentially regulates effector and regulatory $\mathrm{T}$ cell lineage commitment. Immunity 30: 832-844, 2009.

36. Pollizzi KN and Powell JD: Regulation of T cells by mTOR: The known knowns and the known unknowns. Trends Immunol 36: $13-20,2015$. 
37. Lee K, Gudapati P, Dragovic S, Spencer C, Joyce S, Killeen N Magnuson MA and Boothby M: Mammalian target of rapamycin protein complex 2 regulates differentiation of Th1 and Th2 cell subsets via distinct signaling pathways. Immunity 32: 743-753, 2010.

38. Delgoffe GM, Pollizzi KN, Waickman AT, Heikamp E, Meyers DJ, Horton MR, Xiao B, Worley PF and Powell JD: The kinase mTOR regulates the differentiation of helper $\mathrm{T}$ cells through the selective activation of signaling by mTORC1 and mTORC2. Nat Immunol 12: 295-303, 2011.

39. Sarbassov DD, Ali SM, Sengupta S, Sheen JH, Hsu PP, Bagley AF, Markhard AL and Sabatini DM: Prolonged rapamycin treatment inhibits mTORC2 assembly and Akt/PKB. Mol Cell 22: 159-168, 2006.

40. Askar M: T helper subsets and regulatory T cells: Rethinking the paradigm in the clinical context of solid organ transplantation. Int J Immunogenet 41: 185-194, 2014.

41. Pan F, Barbi J and Pardoll DM: Hypoxia-inducible factor 1: A link between metabolism and $\mathrm{T}$ cell differentiation and a potential therapeutic target. Oncoimmunology 1: 510-515, 2012.

42. Dang EV, Barbi J, Yang HY, Jinasena D, Yu H, Zheng Y, Bordman Z, Fu J, Kim Y, Yen HR, et al: Control of T(H)17/T(reg) balance by hypoxia-inducible factor 1 . Cell 146: 772-784, 2011.

43. Hudson CC, Liu M, Chiang GG, Otterness DM, Loomis DC, Kaper F, Giaccia AJ and Abraham RT: Regulation of hypoxiainducible factor 1alpha expression and function by the mammalian target of rapamycin. Mol Cell Biol 22: 7004-7014, 2002.
44. Sutton TA, Wilkinson J, Mang HE, Knipe NL, Plotkin Z, Hosein M, Zak K, Wittenborn J and Dagher PC: p53 regulates renal expression of HIF-1\{alpha\} and pVHL under physiological conditions and after ischemia-reperfusion injury. Am J Physiol Renal Physiol 295: F1666-F1677, 2008.

45. Nieminen AL, Qanungo S, Schneider EA, Jiang BH and Agani FH: Mdm2 and HIF-1alpha interaction in tumor cells during hypoxia. J Cell Physiol 204: 364-369, 2005.

46. Kaluz S, Kaluzová M and Stanbridge EJ: Does inhibition of degradation of hypoxia-inducible factor (HIF) alpha always lead to activation of HIF? Lessons learnt from the effect of proteasomal inhibition on HIF activity. J Cell Biochem 104: 536-544, 2008.

47. Schmid T, Zhou J, Köhl R and Brüne B: p300 relieves p53-evoked transcriptional repression of hypoxia-inducible factor-1 (HIF-1). Biochem J 380: 289-295, 2004.

48. François M,Romieu-Mourez R,Li M and Galipeau J: Human MSC suppression correlates with cytokine induction of indoleamine 2,3-dioxygenase and bystander M2 macrophage differentiation. Mol Ther 20: 187-195, 2012.

49. Mercalli A, Calavita I, Dugnani E, Citro A, Cantarelli E, Nano R, Melzi R, Maffi P, Secchi A, Sordi V, et al: Rapamycin unbalances the polarization of human macrophages to M1. Immunology 140: 179-190, 2013. 